\title{
MICROSTRUCTURE-SENSITIVE FATIGUE MODELING OF MEDICAL-GRADE FINE WIRE
}

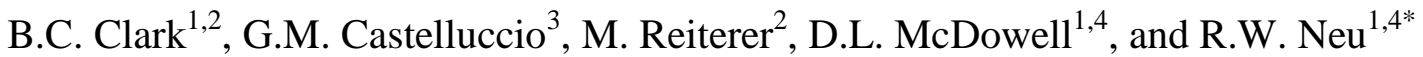 \\ ${ }^{1}$ Woodruff School of Mechanical Engineering, Georgia Institute of Technology, Atlanta, GA, \\ USA \\ ${ }^{2}$ Medtronic, PLC, Minneapolis, MN, USA \\ ${ }^{3}$ School of Aerospace, Transport and Manufacturing, Cranfield University, Cranfield, UK \\ ${ }^{4}$ School of Materials Science and Engineering, Georgia Institute of Technology, Atlanta, GA, \\ USA \\ *corresponding author - Tel: 1-404-894-3074, Email: rick.neu@ gatech.edu
}

\begin{abstract}
This work presents a modeling methodology to assess the sensitivity to microstructure in highcycle fatigue (HCF) performance of fine wires made from MP35N alloy (35Ni-35Co-20Cr10Mo in wt \%) used as conductors in cardiac leads. The model consists of a microstructure generator that creates a mesh of a statistically representative microstructure, a finite element analysis using a crystal plasticity constitutive model to determine the local response behavior of the microstructure, and a post processer employing fatigue indicating parameters (FIPs) to assess the likelihood of fatigue crack initiation. The fatigue crack initiation potency for selected microstructure attributes, boundary and interface conditions, and loading profiles is determined by computing the Fatemi-Socie FIP over a physically-relevant volume of scale. Case studies are used to investigate (1) the influence of non-metallic inclusion proximity to the wire surface on fatigue potency and (2) the transition life demarcating lives primarily controlled by fatigue crack initiation versus microcrack fatigue growth.
\end{abstract}


Keywords: fatigue indicator parameters, crystal plasticity, inclusions, fine wire

\section{NOMENCLATURE}

$k^{*}=$ scaling parameter in Fatemi-Socie for influence of normal stress acting on critical plane

$A=$ anisotropy ratio

$b=$ Burgers vector

$c_{g r}=$ material parameter in threshold strength relation

$C_{\chi}=$ coefficient in back stress evolution

$C_{11}, C_{12}, C_{44}=$ components of elasticity tensor for cubic system

$d_{g}=$ scaling parameter associated with the microstructural size

$d_{g r}=$ nominal grain size

$D^{(\alpha)}=$ drag stress on slip system $\alpha$

$D_{o}=$ drag strength

$E=$ Young's modulus for isotropic material

$f_{t w}=$ twin volume fraction

$F_{G E V}=$ Generalized Extreme Value (GEV) distribution function

$F_{\text {Gumbel }}=$ Gumbel $($ Type I GEV) distribution function

$h_{o}=$ component in latent hardening coefficient matrix

$h^{(\alpha \beta)}=$ latent hardening coefficient matrix

$H=$ height of $\mathrm{AV}$

$k_{1}, k_{2}=$ parameters in dislocation evolution relation

$k_{3}=$ coefficient scaling dislocation density in mean free path relation 
$m=$ number of instantiations conducted at a given $S_{a}$

$n=$ flow exponent

$n_{k}=$ material parameter in threshold strength relation

$N_{f}=$ cycles to failure

$N_{\text {inc }}=$ number of cycles to incubate a fatigue crack

$N_{t}=$ transition life

$P_{F S}=$ Fatemi-Socie parameter

$\tilde{P}_{F S}=$ median of the EV FS values

$\Delta P_{F S}=$ range of EV FS parameter $\left(=\max \left(P_{F S}\right)-\min \left(P_{F S}\right)\right)$

$R=$ stress ratio $\left(=S_{\min } / S_{\max }\right)$

$S_{\min }=$ minimum stress

$S_{\text {max }}=$ maximum stress

$S_{a}=$ stress amplitude $\left(=\left(S_{\max }-S_{\min }\right) / 2\right.$

$t=$ twin spacing

$t_{1}, t_{2}, t_{3}=$ different variants of thicknesses

$T=$ thickness of $\mathrm{AV}$

$W=$ width of $\mathrm{AV}$

$x=$ value in GEV distribution function

$x_{\text {surf }}=$ NMI depth from wire surface

$\alpha_{g}=$ correlation coefficient in Tanaka-Mura relation

$\alpha, \beta=$ slip system indices

$\alpha_{t}=$ coefficient in Taylor hardening relation

$\chi^{(\alpha)}=$ back stress on slip system $\alpha$ 
$\delta^{(\alpha \beta)}=$ Kronecker delta

$\gamma^{(\alpha)}=$ inelastic shear strain on slip system $\alpha$

$\dot{\gamma}_{o}=$ shear strain rate coefficient

$\Delta \gamma_{\max }^{p}=$ maximum plastic shear strain range on the critical plane

$\eta=$ hardening coefficient in back stress evolution

$\eta_{o}=$ scaling parameter for hardening coefficient in back stress evolution

$\kappa^{(\alpha)}=$ threshold stress on slip system $\alpha$

$\kappa_{o}^{(\alpha)}=$ initial critical resolved shear stress on slip system $\alpha$

$\Lambda^{(\alpha)}=$ mean free path for dislocation motion of slip system $\alpha$

$\mu=$ shear modulus resolved on slip system

$\mu=$ location parameter in GEV distribution function

$v=$ Poisson's ratio for isotropic material

$\rho^{(\alpha)}=$ dislocation density of slip system $\alpha$

$\rho_{o}=$ initial dislocation density

$\sigma=$ scale parameter in GEV distribution function

$\sigma_{n}^{\max }=$ maximum stress normal to the critical plane

$\sigma_{y}=$ yield strength

$\tau^{(\alpha)}=$ shear stress on slip system $\alpha$

$\tau_{o}^{(\alpha)}=$ lattice resistance of slip system $\alpha$

$\Omega_{F S}=$ weighted EV FS variability parameter

$\xi=$ shape parameter in GEV distribution function 


\section{ABBREVIATIONS}

$35 \mathrm{~N}$ LT $=$ low $\mathrm{Ti}$ version of MP35N (registered trademark of Fort Wayne Metals Research Products Corporation, Fort Wayne, IN, USA)

$\mathrm{AV}=$ averaging volume

$\mathrm{CDF}=$ cumulative density function

$\mathrm{CPFEM}=$ crystal plasticity finite element method

$\mathrm{EV}=$ extreme value

FIP = Fatigue ilIndicator Parameter

$\mathrm{FS}=$ Fatemi-Socie

$\mathrm{GEV}=$ generalized extreme value

$\mathrm{HCF}=$ high cycle fatigue

ISV = internal state variable

MFP $=$ mean free path

MP35N = alloy of 35Co-35Ni-20Cr-10Mo in wt \% (registered trademark of SPS Technologies, Jenkintown, PA, USA)

$\mathrm{NMI}=$ non-metallic inclusions

$\mathrm{RBBF}=$ rotating beam bending fatigue

$\mathrm{SVE}=$ statistical volume element

$\mathrm{T}-\mathrm{M}=$ Tanaka-Mura

$\mathrm{TEM}=$ transmission electron microscope

$\mathrm{TTF}=$ tension-tension fatigue

UMAT $=$ user material subroutine for finite element code, ABAQUS

$\mathrm{VHCF}=$ very high cycle fatigue 


\section{INTRODUCTION}

A robust understanding of component fatigue behavior is critical for medical devices, especially for permanently implantable, life sustaining applications in which minimizing invasive procedures and interventions is highly desirable. In the case of cardiac pacing leads, the in-situ loading conditions are variable and difficult to quantify. Heart contractions create a lowamplitude, high-frequency load, and torso and arm movements add higher amplitude, but low frequency loading. In the high cycle fatigue (HCF) regime, the fatigue life of fine wires is dominated by fatigue crack initiation, defined here as nucleation and growth of a crack on the order of the nucleant grain/phase size. Once formed, a fatigue crack grows quickly to reach the critical crack length due to the geometric constraints of the wire, after which ductile (fast) fracture occurs. Fatigue crack nucleation in fine wires is a stochastic process often controlled by heterogeneities within the microstructure such as non-metallic inclusions (NMIs). Understanding the role these inclusions play in fatigue life variability is critical to the design of fatigue resistant lead wires.

Past studies [20] on MP35N medical grade wires have employed statistical Monte Carlo initiation life models to predict materials intrinsic variability. However, these models are constrained by a limited capability to represent the microstructure of these wires. Microstructure-sensitive fatigue models are attempts to represent scatter in fatigue life by explicitly considering the effects of microstructure [N1,N2,N3,N4]. Among the microstructure attributes that explain most of the variability are grain size and distribution of crystallographic orientations (i.e., crystallographic texture), phase volume fraction and distribution, the 
interactions among adjacent grains, grain boundary character, non-metallic inclusion attributes, inclusion/matrix interface behavior, and voids or other pre-existing heterogeneity at the scale of the microstructure. By incorporating the statistical variability of microstructure attributes into the model, the fatigue life distribution of a component can be estimated from a limited set of fatigue experiments.

The major components of a microstructure-sensitive fatigue model involve:

1. A representation of one or more microstructural attributes that vary in conformance to some prescribed distributions informed from experiments;

2. A method for applying representative fatigue loading and tracking the evolution of local stresses and strains;

3. A metric to evaluate fatigue damage potency, i.e., a Fatigue Indicator Parameter (FIP) [N3]. This involves combining key response parameters in a manner that provide an indication of the fatigue damage potency of the applied loading considering the microstructure attributes represented.

Using a crystal plasticity finite element model (CPFEM) governed by a set of constitutive laws, different microstructural attributes can be modeled and quickly assessed for their impact on fatigue including slip localization, plastic strain heterogeneity due to geometrical features (notches, etc.) and grain-to-grain and grain-to-inclusion interactions. Specific applications of microstructure-sensitive fatigue modeling considered in recent work include:

1. Establishing rankings of microstructure attributes most detrimental to life using extreme value marked correlation functions $[\mathrm{N} 3, \mathrm{~N} 5,16]$;

2. Establishing the effect of the loading amplitude on fatigue scatter [11, 18,GMC1];

3. Determining the influence of geometrical features on fatigue resistance $[10,11$, 


\section{GMC1];}

4. Predicting fatigue scatter due to the attributes of the primary inclusion particles which may include the inclusion size and type, the influence of the interface between the inclusion and matrix (e.g., perfectly bonded, partially debonded, fully debonded), the polycrystalline microstructure around the inclusion, and proximity of the inclusion to the surface or other inclusions [N6, 18];

5. Understanding the competing high cycle fatigue mechanisms for surface vs. subsurface crack nucleation [N7].

Although Schaffer [19] developed a numerical model for fine wire MP35N incorporating the influence of several microstructural inputs via Monte Carlo methods, his model does not account for polycrystalline microplasticity which strongly contributes to $\mathrm{HCF}$ crack nucleation through localized anisotropic deformations [N1, N2, N3,GMC2]. The objective of this work is to develop a microstructure-sensitive fatigue simulation for $\mathrm{MP} 35 \mathrm{~N}$ fine wire capable of elucidating differences in fatigue performance due to variability of microstructure attributes. This modeling strategy includes [N1]:

1. Formulation of crystal plasticity relations that capture the rate sensitivity and kinematic hardening behavior of MP35N fine wire;

2. Development of a microstructure generation and meshing protocol to recreate salient MP35N microstructure attributes in a stochastic, finite-element framework;

3. Selection of appropriate response parameters to assess fatigue performance;

4. Characterization of the extreme-value distributions of the selected response parameters; and

5. Validation of the newly-developed CPFEM model against experimental data. 


\section{Fine Wire Material}

The fine wires are made from MP35N (ASTM F562) [2], a quaternary, low temperature superalloy containing major elements (wt \%) 35Ni-35Co-20Cr-10Mo. The high amount of Ni produces a metastable face-centered cubic crystal structure. The fine wire form of MP35N has found use in surgical implants due its excellent corrosion resistance and biocompatibility [13] as well as its high strength and fatigue resistance. Applications include catheters, stylets and pacing leads.

Production of wires is accomplished by drawing a rod through successively smaller dies with intermediate annealing steps. The drawing process produces significant anisotropy in the material with strong texture components in the $\langle 001\rangle,\langle 111\rangle$ and $\langle 113\rangle[7,14,23]$. Drawing also contributes to a fine grain structure. Grain size for fine wire is typically $1-5 \mu \mathrm{m}$, compared with $35 \mu \mathrm{m}$ or greater for the bulk material. In the bulk material, hexagonal close packed platelets form through the Suzuki mechanism $[1,5]$. These platelets have not been observed in fine wire specimens $[14,23]$ or bulk specimens under room-temperature deformation [17], leading to its characterization as a single-phase material. Plastic deformation is accommodated through both slip and intragranular twinning [23]. Twins are found to be between 1-10 $\mathrm{nm}$ in thickness. Once formed, deformation twins also act as a hardening mechanism, impeding the motion of dislocations.

The presence of non-metallic inclusions (NMIs) is a primary driver of fatigue crack initiation in MP35N wires [19,20]. Two types of inclusion particles have been identified, shown in Fig. 1: cuboidal titanium nitride (TiN) and globular aluminum oxide $\left(\mathrm{Al}_{2} \mathrm{O}_{3}\right)$. The former particles are typically larger in size $(4-10 \mu \mathrm{m})$ compared to the later $(1-5 \mu \mathrm{m})$. A related alloy 
designated $35 \mathrm{~N}$ LT is a low $\mathrm{Ti}$ variant where $\mathrm{Ti}$ is reduced below $0.01 \%$ to eliminate $\mathrm{TiN}$ particles, improving fatigue performance [19,20]. In this work, the terms full-Ti or low-Ti will be used to differentiate between the MP35N or 35N LT variants when necessary.
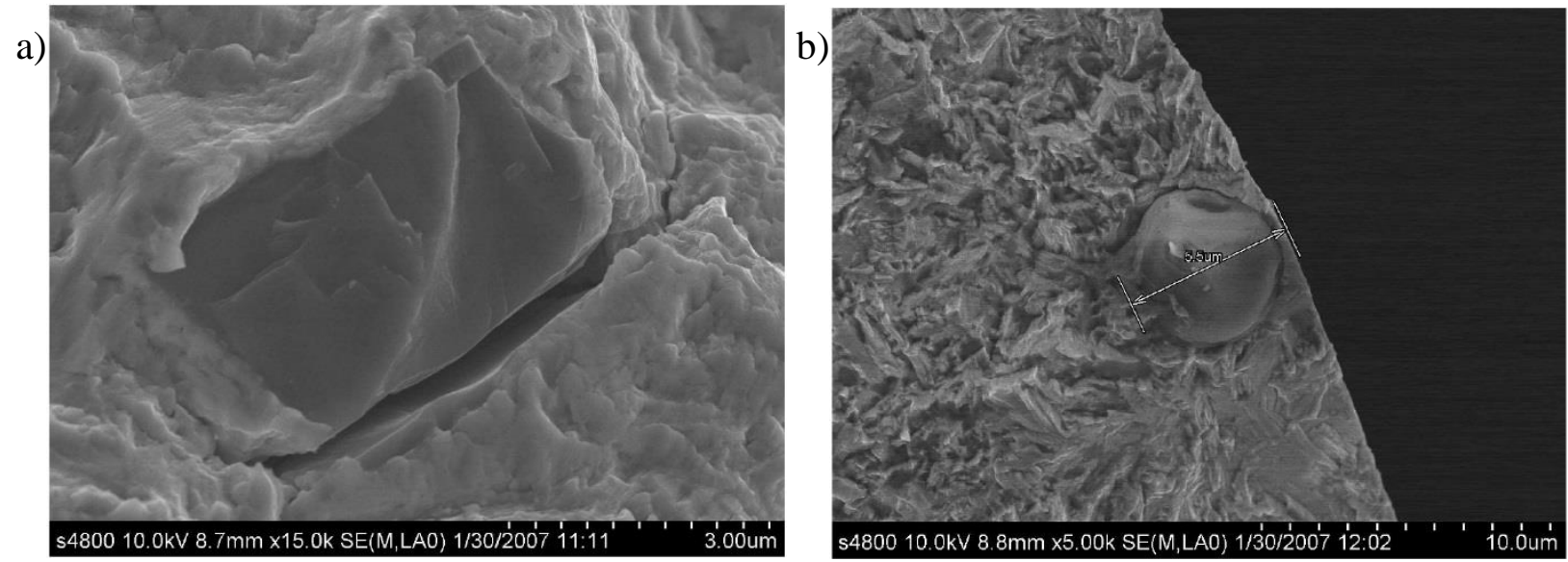

Fig. 1 Inclusions in MP35N fine wire. (a) Sharp cuboidal TiN inclusion, partially debonded from the matrix. (b) Globular A12O3 (alumina) inclusion near the wire surface. Note differences in scale. From Ref. [19]

\section{Characterization of Microstructure Attributes}

The salient microstructure attributes of the MP35N fine wire, characterized by Prasad et al. [14], provide realistic input for virtual microstructure instantiation. Grain size and texture distributions were characterized via electron back scatter diffraction (EBSD) imaging of a transverse wire cross-section. Grain morphology was characterized with their aspect ratio, i.e., by comparing longitudinal and transverse EBSD cross-sections. The MP35N fine wire has a grain size to be on the order of 1-5 $\mu \mathrm{m}$ with variation following a lognormal distribution. MP35N in its cold-drawn condition exhibits a strong fiber texture produced from the wire drawing, shown in Fig. 2. 

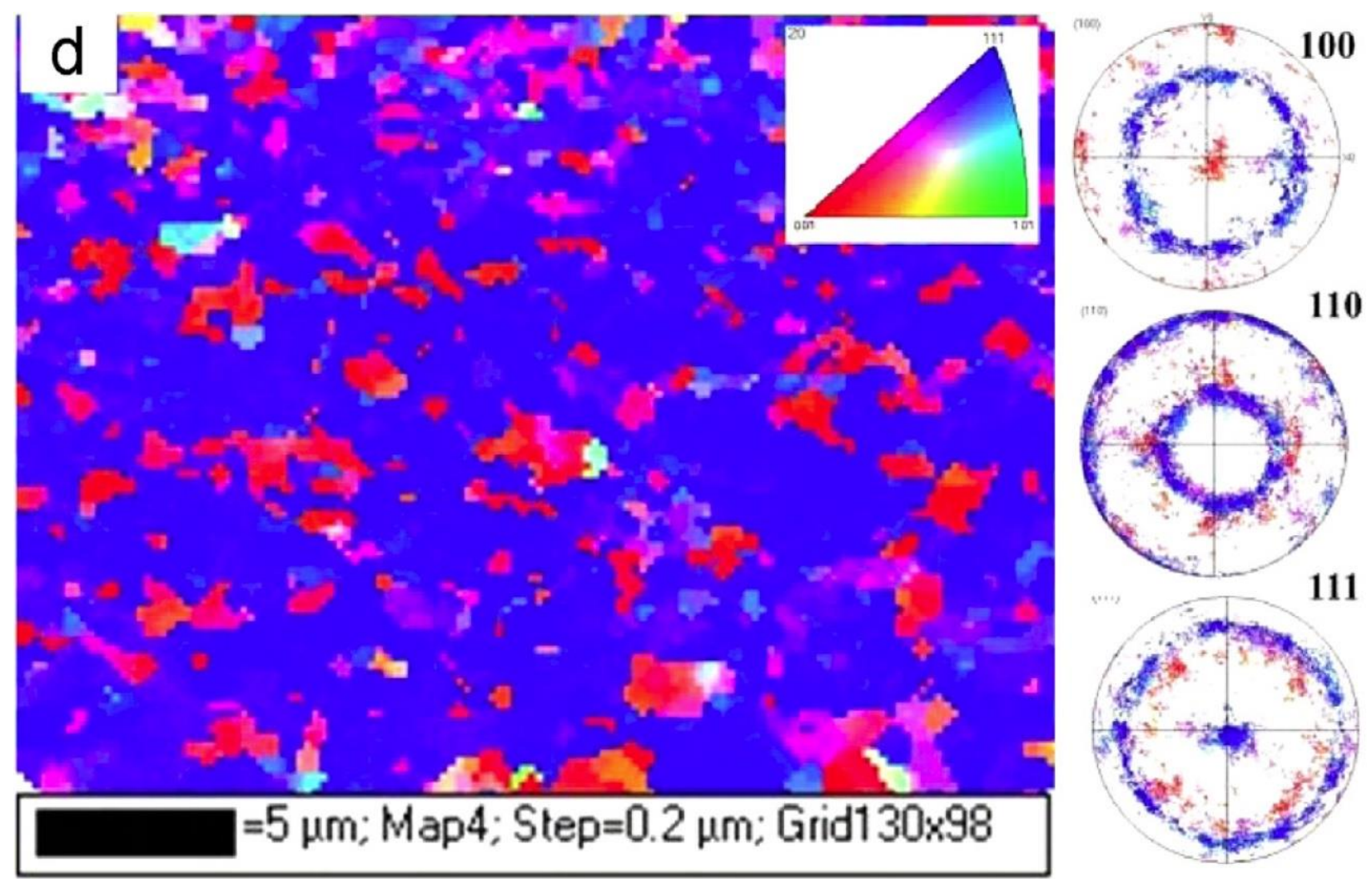

Fig. 2 An inverse pole figure map of low-Ti MP35N fine wire microstructure created by EBSD accompanied by pole figures showing strong $\langle 111\rangle$ texture along axis of wire. From Ref. [14].

\section{Modeling Methodology}

The modeling methodology consists of several components that will be described in more detail in the following sections:

1. A microstructure generation tool that creates a finite element mesh of a stochastic arrangement of grains (a so-called instantiation of microstructure), often surrounding a NMI within the defined volume;

2. A finite element solver (ABAQUS) coupling to crystal plasticity model for MP35N to solve for the local cyclic stress and strain states generated under the simulated fatigue loading;

3. A post-processer to compute the local volume-averaged response parameters (FIPs) 
from each microstructure instantiation;

4. The extreme-value FIP (Fatemi-Socie (FS) parameter in this case) distribution, populated from the maximum FS value of each microstructure instantiation, are then correlated to the distribution of fatigue lives to provide a direct quantitative comparison to the experimental fatigue data.

\section{Volume Elements Representing the Microstructure}

To model the stochastic nature of metallic microstructures in a computationally feasible way, it is useful to employ Statistical Volume Elements (SVEs) [N3,N9,N10]. These idealized volumes are constructed such that each identically-sized volume is a sample of the underlying distributions of the microstructure attributes. Each SVE contains a unique, random arrangement of grains and crystallographic textures which are sampled from experimentally characterized grain size and texture distributions. We consider SVE that are large enough relative to the grain size that the average stress-strain responses of multiple SVEs converge to the macroscopic stress-strain response determined by experiment. The use of SVEs for numerical fatigue modeling offers advantages in computational efficiency. A limited number of SVEs $(<100)$ at each loading condition can adequately characterize the distribution of the desired response parameter $[\mathrm{N} 5,16]$. Variation of microstructure attributes between successive SVEs results in differences in the local stress-strain response. These differences can be quantified using FIPs which are surrogate measures of the local driving force for fatigue crack nucleation and early growth.

Each volume element is created using an ellipsoid packing algorithm [16] and uses a meshing algorithm based on Musinski's work [10]. The target microstructure is a small volume 
of a MP35N fine-wire matrix surrounding a cuboidal TiN inclusion particle. Since the goal of the model is to examine rare event phenomenon associated with NMIs, an inclusion is input deterministically to each instantiation with full control of inclusion size, position and interface. The loading, interface and boundary conditions around the NMI can all be manipulated to examine their effect on fatigue potency. The term fatigue potency relates to the likelihood of fatigue crack initiation as quantified by FIPs.

\section{Constitutive Models}

In the fine wire configuration, MP35N consists of a single-phase, face-centered cubic (fcc) structure with intragranular deformation twins. An elastic-crystal viscoplastic constitutive model established for another fcc alloy [21] was adopted with modification to phenomenologically account for the influence of deformation twins, dependent on twin volume fraction $f_{t w}$ and twin spacing $t$. Homogenization over deformation twins is necessary due to the limited spatial resolution of finite element modeling. The model seeks to predict damage processes at the scale of microns, while deformation twins have thicknesses of the order of 1 to $10 \mathrm{~nm}[14,23]$.

The inelastic shear strain rate on each slip system $\alpha$ is given by a power law relationship,

$$
\dot{\gamma}^{(\alpha)}=\dot{\gamma}_{o}\left\langle\frac{\left|\tau^{(\alpha)}-\chi^{(\alpha)}\right|-\kappa^{(\alpha)}}{D^{(\alpha)}}\right\rangle^{n} \operatorname{sgn}\left(\tau^{(\alpha)}-\chi^{(\alpha)}\right)
$$

that depends on the shear stress $\tau^{(\alpha)}$, the back stress $\chi^{(\alpha)}$, which is critical for modeling the cyclic loading and ratchetting, the threshold stress $\kappa^{(\alpha)}$, and drag stress $D^{(\alpha)}$ acting on each slip system. In addition, $\dot{\gamma}_{o}$ is the shear strain rate constant and $n$ is the flow exponent. The inelastic shear strain rate is zero on each slip system until the threshold stress $\kappa^{(\alpha)}$ is reached. Plastic slip is assumed to occur only on the 12 octahedral systems $\{111\}\langle 110\rangle$. 
The threshold hardening equation depends on dislocation density $\rho$ through a Taylor relation

$$
\kappa^{(\alpha)}=\kappa_{o}^{(\alpha)}+\alpha_{t} \mu b \sqrt{\rho^{(\alpha)}}
$$

where $b$ is the Burgers vector, $\mu$ is the (resolved) shear modulus, $\alpha_{t}$ is a constant and $\kappa_{o}$ is the initial critical resolved shear stress (CRSS) given by

$$
\kappa_{o}^{(\alpha)}=\left[\left(\tau_{o}^{(\alpha)}\right)^{n_{k}}+c_{g r}\left(d_{g r}\right)^{-0.5}+c_{g r}\left(f_{t w}\right)\right]^{1 / n_{k}}
$$

which depends on the lattice resistance $\tau_{o}$, the nominal grain size $d_{g r}$, and the twin volume fraction $f_{t w}$ as well as material parameters $c_{g r}$ and $n_{k}$.

Two internal state variables (ISVs) can evolve: dislocation density and back stress. Dislocation density evolves by

$$
\dot{\rho}^{(\alpha)}=\sum_{\beta=1}^{12} h^{(\alpha \beta)}\left(\frac{k_{1}}{b \Lambda^{(\beta)}}-k_{2} \rho^{(\beta)}\right)\left|\dot{\gamma}^{(\beta)}\right|
$$

where $h^{(\alpha \beta)}$ is the hardening coefficient matrix, $\Lambda$ is the mean free path (MFP) for dislocation motion, and $k_{1}$ and $k_{2}$ are constants. The dislocation density affects both isotropic and kinematic hardening. At high dislocation densities typical of strongly cold-worked components, competition between dislocation formation and annihilation results in saturation of $\rho$ due to the dynamic equilibrium between the first and second terms of Eq. 4. Due to the low stacking fault energy (SFE) of MP35N, it is assumed that there is no interaction between the slip systems and only self-hardening is possible; indeed, self-hardening can even dominate on fcc metals with higher SFE [GMC3]. Therefore, the hardening coefficient matrix is given by

$$
h^{(\alpha \beta)}=h_{o} \delta^{(\alpha \beta)}
$$

where $h_{o}$ is a constant and $\delta^{(\alpha \beta)}$ is the Kronecker delta. The MFP $\Lambda$ is a measure of the obstacle-free movement distance available to a dislocation on a given slip system. In MP35N, it 
is described by the harmonic mean of three distances: the grain size $d_{g r}$, twin spacing $t$, and the spacing of immobile dislocations which scales inversely with the square root of dislocation density,

$$
\frac{1}{\Lambda^{(\beta)}}=\frac{1}{d_{g r}}+\frac{1}{t}+k_{3} \sqrt{\rho^{(\beta)}}
$$

The back stress evolves according to

$$
\dot{\chi}^{(\alpha)}=C_{\chi}\left[\eta \mu b \sqrt{\rho^{(\alpha)}} \operatorname{sgn}\left(\tau^{(\alpha)}-\chi^{(\alpha)}\right)-\chi^{(\alpha)}\right]\left|\dot{\gamma}^{(\alpha)}\right|
$$

where $C_{\chi}$ is a fitting parameter and $\eta$ depends on $d_{g r}$, twin spacing $t$, and $\Lambda$ by the relation

$$
\eta=\eta_{o} \Lambda^{(\alpha)}\left(\frac{1}{d_{g r}}+\frac{1}{t}\right)
$$

The back stress equation contains two terms: an accumulation term that depends on the dislocation density on the current slip system, and a dynamic recovery term dependent on the current value of $\chi^{(\alpha)}$ representing the influence of dislocation annihilation. The back stress ISV captures the Bauschinger effect and plastic ratcheting that occurs under cyclic loading as a result of non-uniform mesoscale structures such as dislocation pile-ups, twin boundaries, and dislocation walls.

The parameters of the constitutive model were calibrated using a combination of existing values from literature, physically-based estimations, and iterative fitting against cyclic mechanical test data conducted on the fine wires. The material response is a function of both the parameter values and the crystallographic texture of the microstructure, so the parameter fits must be adjusted for significant changes in texture. Three distinct model calibrations were performed: an initial calibration that was a first-order approximation targeting a microstructure with a single texture distribution around the wire drawing texture $\langle 111\rangle$, an intermediate calibration to improve the kinematic hardening response, and a fine-tuning calibration targeting a 
microstructure with multiple texture components shown in Fig. 2.

The isotropic hardening behavior and the strain-rate sensitivity were calibrated to uniaxial tensile test data reported in Ref. [14]. This test was conducted on a $250 \mathrm{~mm}$ length of low-Ti as-drawn MP35N wire in displacement control alternating every $0.5 \%$ strain between high and low strain rates of $1.7 \times 10^{-3}$ and $1.7 \times 10^{-5} \mathrm{~s}^{-1}$, respectively. The parameters governing the kinematic hardening behavior were determined by fitting to the model to two cyclic tension-tension force-control experiments, both conducted at $\mathrm{R}=0.5$, with $S_{\max }=1400$ MPa and $1500 \mathrm{MPa}$. These experiments exhibit cyclic plastic ratchetting. The difference in accumulated ratchet strain between the two tests caused by the non-zero mean stress was compared to determine the increment of strain over each cycle which was used to calibrate the back stress evolution parameters.

The material parameters are summarized in Table 1. Parameters $D_{o}, \tau_{o}, \alpha_{t}, b$, $n_{k}, c_{g r}, C_{\chi}$, and $h_{o}$ are unchanged from the values used for IN100 [21]. The values of these parameters for MP35N are expected to be similar to those for IN100, since both are fcc alloys containing significant Ni content. Parameters $d_{g r}, f_{t w}, t$ and $k_{3}$ were added to account for the strengthening effect of the small grain size and nano-scale twins. The value of $d_{g r}$ was set to the median grain size of $2 \mu \mathrm{m}$ and $t$ was given a value of $10 \mathrm{~nm}$ consistent with the twin spacing revealed by TEM [14, 23]. The values of $f_{t w}$ and $k_{3}$ were chosen to reflect physically reasonable values. The initial dislocation density $\rho_{o}$ was given a value reflecting the high initial dislocation density from $36 \%$ cold work in the as-drawn MP35N wire. Additional cyclic deformation from fatigue type loading is not expected to further increase $\rho$, so the ratio of $k_{1}$ and $k_{2}$ is selected such that $\rho$ saturates above $\rho_{o}$.

Since a complete set of the elastic properties, $C_{11}, C_{12}$, and $C_{44}$, was not available for 
MP35N, and experiments could only provide the uniaxial value for a textured wire, density functional theory (DFT) using a calculation methodology derived from the technique in Wang et al. [26] was first used to determine the anisotropy ratio of MP35N,

$$
A=\frac{2 C_{44}}{C_{11}-C_{12}}=1.56
$$

Then it was assumed that this anisotropy ratio predicted at 0 Kelvin is same at room temperature, but scaled to match the experimentally measured modulus of a volume with known crystallographic texture. These values are reported in Table 1. The TiN inclusions were assumed to be isotropic elastic with $E=360,000 \mathrm{MPa}$ and $v=0.24$. The parameters having the largest effect on the stress-strain response of a SVE were the elastic stiffness coefficients, $C_{11}$, $C_{22}$, and $C_{44}$, followed by the rate exponent $n$ and the back stress evolution coefficient $\eta_{o}$ [N11]. However, the life correlations were much more sensitive to the T-M correlation coefficient $\alpha_{g}$ than any of the constitutive model parameters.

Table 1 Parameters used in constitutive model for MP35N.

\begin{tabular}{cc}
\hline Parameter & Value \\
\hline$C_{11}$ & $237,321 \mathrm{MPa}$ \\
$C_{12}$ & $118,120 \mathrm{MPa}$ \\
$C_{44}$ & $92,756 \mathrm{MPa}$ \\
$\dot{\gamma}_{o}$ & $7.2 \times 10^{16} \mathrm{~s}^{-1}$ \\
$n$ & 18 \\
$D_{o}$ & $195 \mathrm{MPa}$ \\
$\tau_{o}$ & $75.15 \mathrm{MPa}$ \\
$\rho_{o}$ & $3.0 \times 10^{9} \mathrm{~mm}{ }^{-2}$ \\
$\alpha_{t}$ & 0.1 \\
$\mu$ & $92,756 \mathrm{MPa}$ \\
$b$ & $0.407 \mathrm{~nm}$ \\
$n_{k}$ & 1
\end{tabular}




\begin{tabular}{cc}
$c_{g r}$ & $9.432 \mathrm{MPa} \sqrt{m m}$ \\
$d_{g r}$ & $0.002 \mathrm{~mm}$ \\
$f_{t w}$ & 0.1 \\
$C_{\chi}$ & 2 \\
$\eta_{o}$ & 68.0 \\
$t$ & $0.00001 \mathrm{~mm}$ \\
$h_{o}$ & 1 \\
$k_{1}$ & $100,000 \mathrm{~mm}^{-1}$ \\
$k_{2}$ & 1.0 \\
$k_{3}$ & 0.1 \\
\hline
\end{tabular}

\section{Fatigue Indicator Parameters}

Fatigue Indicator Parameters (FIPs) provide a way to determine the location and relative potency of fatigue hot-spots within a component after the application of fatigue loading [N3]. FIPs are physically-based metrics that combine tensor quantities such as stresses or plastic strains occurring over a representative load cycle into a single scalar value which can be used to judge the relative fatigue potency. Numerous FIPs have been proposed and utilized for different materials and crack nucleation mechanisms.

The Fatemi-Socie (FS) parameter [6] was selected for use with the model for its ability to predict fatigue response in materials where crack nucleation is driven by localized cyclic shear strain. The parameter is based on the observation that cyclic fatigue cracks tend to form on planes aligned with the direction of maximum shear strain amplitude, but that magnitude of shear strain amplitude alone does not explain the lower rates of cracking in torsional fatigue compared to uniaxial. To account for this, the maximum plastic shear strain amplitude over a cycle is modified by the normal stress to the plane of maximum plastic shear strain. The FS parameter is 
given by

$$
P_{F S}=\frac{\Delta \gamma_{\max }^{p}}{2}\left[1+k^{*} \frac{\sigma_{n}^{\max }}{\sigma_{y}}\right]
$$

where $\Delta \gamma_{\max }^{p}$ is the maximum range of plastic shear strain on the critical plane over a cycle and $\sigma_{n}^{\max }$ is the maximum stress normal to the critical plane. The maximum normal stress is normalized by the yield stress $\sigma_{y}$ and weighted by the coefficient $k^{*}$. The weighting coefficient can be estimated by correlating uniaxial to torsional fatigue data. Lacking torsional data for MP35N fine wire, $k^{*}$ has been arbitrarily set to 1 , which is within the range of values found in fatigue literature [3, 11, GMC4]. The FS parameter as formulated in Eq. 10 is termed a critical plane type FIP since it accounts for preferential crack nucleation on cyclic shear planes, either crystallographic [GMC5] or non-crystallographic [GMC4, N8]. The crystallographic formulation finds the critical plane by searching among all available slip systems, while the noncrystallographic formulation takes the plane of maximum cyclic shear strain in 3D space. The non-crystallographic formulation is used in this work to simplify computation. The choice of critical plane calculation methodology is not expected to significantly impact the parameter scaling.

The FS parameter must be evaluated over an appropriate volume to provide a meaningful indication of fatigue crack nucleation potency. Two important considerations for averaging volume (AV) selection are size and sampling location within the SVE. Volume size is dictated by (a) the finite size of fatigue crack initiation, (b) regularization to eliminate mesh-size dependency and (c) desired level of smoothing over microstructural features such as grains. The term initiation is not well-defined in literature, having no single agreed-upon criteria. For the purposes of this research, a fatigue crack is considered initiated when the cracked area within the matrix approaches $1 \mu \mathrm{m}^{2}$. This size was chosen to match the scale of grains in MP35N fine wire, 
which is the smallest feature explicitly modeled in the FEA mesh. Therefore, the size of the AVs used will be of this same scale. Sampling location is associated with the locations of stress risers within the microstructure which provide the driving force for crack initiation. In many cases, the locations of stress risers are unknown a priori so the entire SVE must be interrogated to locate them. However, when a hard NMI is present within the SVE, stress concentrations will occur along the inclusion-matrix interface, permitting a targeted application of sampling locations there. Inclusions that are half debonded from the matrix in an orientation perpendicular to the loading axis will generate their maximum stresses along the debonding perimeter [18,N6]. Under HCF conditions, stresses quickly approach their far-field values moving radially outward away from the NMI surface, resulting in insufficient driving force to generate plasticity more than a few microns from the NMI interface. Because of this, AVs are sampled immediately adjacent to the NMI. These sampling locations for the 50\% debonded NMI configuration have been shown in prior work $[18, N 6]$ to be the locations of the largest FIP magnitude. Fig. 3 illustrates the locations of selected FIP AVs for the case of a 50\% debonded TiN inclusion.

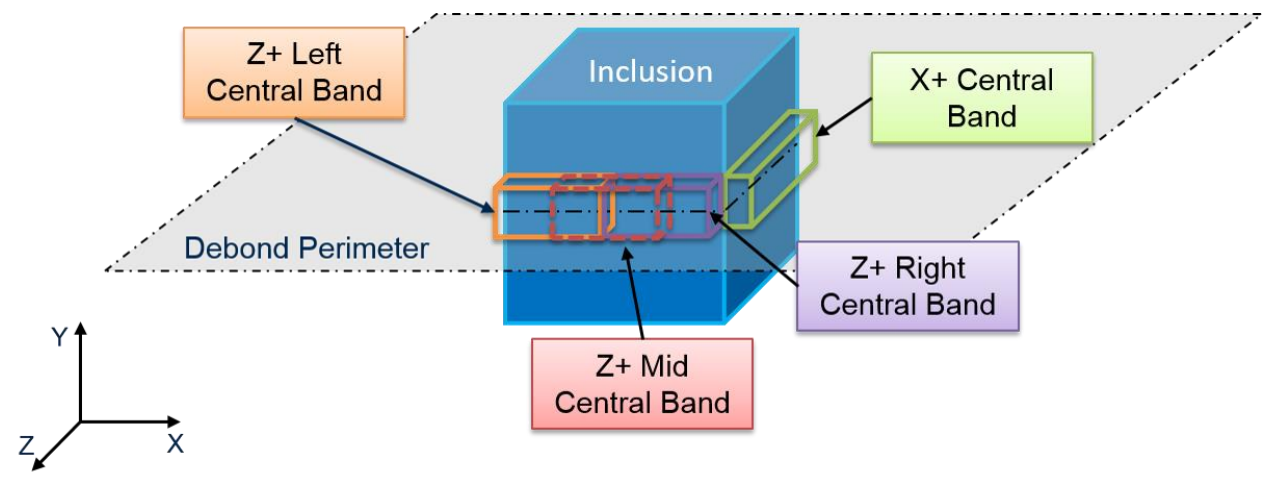

Fig. 3 Schematic showing the positioning and naming conventions of selected FS AVs with respect to a $50 \%$ debonded cuboidal inclusion.

Each of the four distinct volumes shown is replicated on the $X+, X-, Z+$ and $Z-$ inclusion 
faces. The plane labeled Debond Perimeter bisects the inclusion along the Y-axis. Matrix elements above this plane are debonded from the inclusion surface by means of a frictionless normal contact, while elements below are bonded via tie constraints. All FIP AVs are bisected by the debond perimeter such that they contain both bonded and debonded elements in equal measure. Each volume is a rectangular prism of dimensions $W \times H \times T$ where $W$ is the width measured in the plane of the debond perimeter, $H$ is the height along the $\mathrm{Y}$-axis and $T$ is the AV thickness measured radially away from the inclusion and perpendicular to the inclusion face. Each AV has $H=1 \mu \mathrm{m}$ and comes in three variants of thickness, $0.10 \mu \mathrm{m}, 0.25 \mu \mathrm{m}$, and 0.50 $\mu \mathrm{m}$, measured perpendicular to the inclusion face. Two widths of AV were considered. Domains denoted by Full Face span the width of the inclusion face, while domains Left, Right, and Mid have a width equal to half the inclusion width. The Mid domain overlaps both the Left and Right domains by half. Table 2 gives the volumes of each AV in $\mu \mathrm{m}^{3}$ for the case of a $4 \mu \mathrm{m}$ NMI. In total, considering the four edges of the cubic NMI, 48 distinct AVs are defined.

Table 2 Volumes (in $\mu m^{3}$ ) of the FIP AVs for a cubic NMI with edge length of $4 \mu m$ for each of the three variants of thickness.

\begin{tabular}{llll}
\hline AV Identifier & $t_{1}=0.10 \mu \mathrm{m}$ & $t_{2}=0.25 \mu \mathrm{m}$ & $t_{3}=0.50 \mu \mathrm{m}$ \\
\hline Full Face & 0.4 & 1.0 & 2.0 \\
Left Face & 0.2 & 0.5 & 1.0 \\
Mid Face & 0.2 & 0.5 & 1.0 \\
Right Face & 0.2 & 0.5 & 1.0 \\
\hline
\end{tabular}

\section{Extreme Value Statistics}


Statistics of extreme values (i.e., maxima and minima) are useful in the study of the fatigue behavior of engineering components [N6, N4, N3, N5, 16]. Engineering components used in life critical applications must be designed to make the likelihood of fatigue failure extremely small. Prediction of reliability requires characterization of the behavior of the tail end of the population which fails prior to its designed lifespan. Extreme value statistics characterize this tail. Three classes of extreme-value distributions - Gumbel (Type I), Fréchet (Type II) and Weibull (Type III) - can be described by a single distribution through the addition of a shape parameter [18]. This combined distribution is known as the Generalized Extreme Value (GEV) distribution. The cumulative distribution function (CDF) for the GEV distribution is given by

$$
F_{G E V}(x ; \mu, \sigma, \xi)=e^{-\left[1+\xi\left(\frac{x-\mu}{\sigma}\right)\right]^{-1 / \xi}}
$$

where $\mu$ is the location parameter, $\sigma$ is the scale parameter and $\xi$ is the shape parameter. Parameters $\mu$ and $\sigma$ are permitted to be any real number, but $\xi$ is restricted to the interval $[-1,1]$. The shape parameter significantly alters the behavior of the GEV distribution depending on whether $\xi>0, \xi=0$, or $\xi<0$. In the case of $\xi=0$, Eq. 11 is undefined and must be replaced by the limit as $\xi \rightarrow 0$ resulting in

$$
F_{\text {Gumbel }}(x ; \mu, \sigma, \xi)=e^{-e^{\left(-\frac{x-\mu}{\sigma}\right)}}
$$

also known as the Gumbel or Type I GEV distribution. In this work, the GEV distribution (Eq. 11) is used to fit the distributions of the volume-averaged FS parameter and the corresponding fatigue life correlations. Several distribution fits were evaluated and it was found that the fit to the Gumbel distribution was much worse than the GEV distribution [N11].

\section{Correlation to Life}

Once a sufficiently large sample of the extreme-value FS response values has been 
constructed from multiple microstructure instantiations, the sample can then be correlated to a life distribution using a modified Tanaka-Mura (T-M) approach [25] [3]. The Tanaka-Mura equation considers that the number of cycles required to initiate a crack along a slip band under $\mathrm{HCF}$ loading is related to the energy required to form new surfaces which is inversely proportional to the square of the cyclic plastic shear strain range. By substituting the extremevalue FS parameter for cyclic plastic shear strain range, the following relation emerges [22]:

$$
N_{i n c}=\frac{\alpha_{g}}{d_{g}}\left(P_{F S}\right)^{-2}
$$

where $N_{\text {inc }}$ is the number of cycles required to initiate a fatigue crack, $d_{g}$ is a scaling parameter associated with the microstructural size scale and $\alpha_{g}$ is a correlation coefficient, determined by fitting the extreme-value FS distribution to an experimental life distribution.

Under HCF and VHCF conditions the total cycles to failure is large, and the great majority of these contribute to crack initiation. Therefore, we neglect the contribution of propagation life to the total life in MP35N fine wire fatigue. The second case study discussed in the next section investigates this assumption further.

\section{RESULTS AND DISCUSSION}

\section{Effect of Inclusion Proximity to Surface}

A parametric study was conducted to investigate the ability of the model to predict the effect of NMI surface proximity on fatigue life. The main objective was to reproduce the trend found by Schaffer [20] regarding the influence of inclusion surface proximity on full-Ti MP35N wire fatigue life. The NMI were placed at four different depths from the wire surface, 0.75, 1.5, 2.0 and $4.0 \mu \mathrm{m}$. Twenty microstructure instantiations were run at each depth to estimate the median fatigue life and scatter. 
Each microstructure instantiation is a $20 \mu \mathrm{m}^{3}$ SVE occupying a volume immediately adjacent to the outer surface of the MP35N wire. The positive X-face is along the wire outer surface, and all other SVE faces are interior. Loading is along the Y axis, coincident with the local wire axis. Periodic boundary conditions are prescribed for the $\mathrm{Y}$ and $\mathrm{Z}$ faces and all edges. The positive $\mathrm{X}$-face is traction-free and unconstrained, while the negative $\mathrm{X}$-face is given a node-wise displacement boundary condition to mimic a periodic boundary. The negative $\mathrm{X}$-face displacement boundary conditions are extracted from a reference analysis having fully 3D boundary conditions but identical mesh and loading history.

Each SVE was instantiated with 1000 grains using the ellipsoid packing method. The grains were given a fiber texture with a single texture component normally distributed about the $\langle 111\rangle$ orientation with standard deviation defined such that $2 \sigma=15^{\circ}$. The NMI depth from the wire surface $x_{\text {surf }}$ was defined as the perpendicular distance from the wire surface to the nearest point of the NMI. Similarly, the centroid distance $x_{c}$ was defined as the perpendicular distance from the wire surface to the NMI centroid. NMI size was fixed at $4 \mu m$ in this study. The NMIs were oriented such that the inclusion faces were parallel to the SVE faces. All NMIs had their upper halves debonded from the matrix, a worse-case scenario.

The loading profile applied to each microstructure instantiation was in the form of three fully-reversed $R=-1$ displacement controlled cycles. This history is intended to replicate loads experienced under rotating beam bending fatigue (RBBF) by a small surface volume. Loading was applied along the global Y-axis, parallel to the wire neutral axis at the bend apex in the RBBF experiments. The magnitude of the loading amplitude applied to each SVE for each NMI depth is based on the far-field stress $S_{Y Y}$ at the NMI centroid, calculated using a linear stress gradient which is a maximum at the wire free surfaces and zero at the neutral axis. 
Twenty microstructure instantiations were run at each of the four NMI depths. The extreme value (EV) (i.e., maximum) FS values from among the twelve Left, Mid or Right Face volumes were plotted versus NMI distance from surface are shown in Fig. 4. The EV FS values plotted in Fig. 4 were correlated to fatigue life values through the modified Tanaka-Mura approach. The correlation to life was performed at the $0.75 \mu \mathrm{m}$ level assuming negligible propagation life. The median EV FS value was correlated to the linear regression fit the experimental data points with $d_{g}=1.5 \mu \mathrm{m}$. A correlation coefficient $\alpha_{g}=1.129 \times 10^{-5} \mu \mathrm{m}-$ cycles was found to correlate well to experiment. The lives for the other NMI depths were predicted using the same values of $\alpha_{g}$ and $d_{g}$. The resulting life correlations are plotted against the experimental data points in Fig. 5. As expected, life increases as the NMI depth from the free surface is increased. Moreover, the model shows good correlation with the linear regression trend computed from the experimental data points at all depths considered. The minimum lives predicted by the model at each level is below the $5 \%$ confidence bound. This indicates that the model gives a more conservative prediction of minimum fatigue life than the regression fit. Similarly, confidence in the minimum life value predicted by the model at each NMI depth could be improved by running additional microstructure instantiations at that condition. The distribution of lives at each NMI depth can be further investigated by examining the empirical CDFs and fitting GEV distributions. The empirical CDFs along with the fitted GEV distributions are plotted in Fig. 6. 


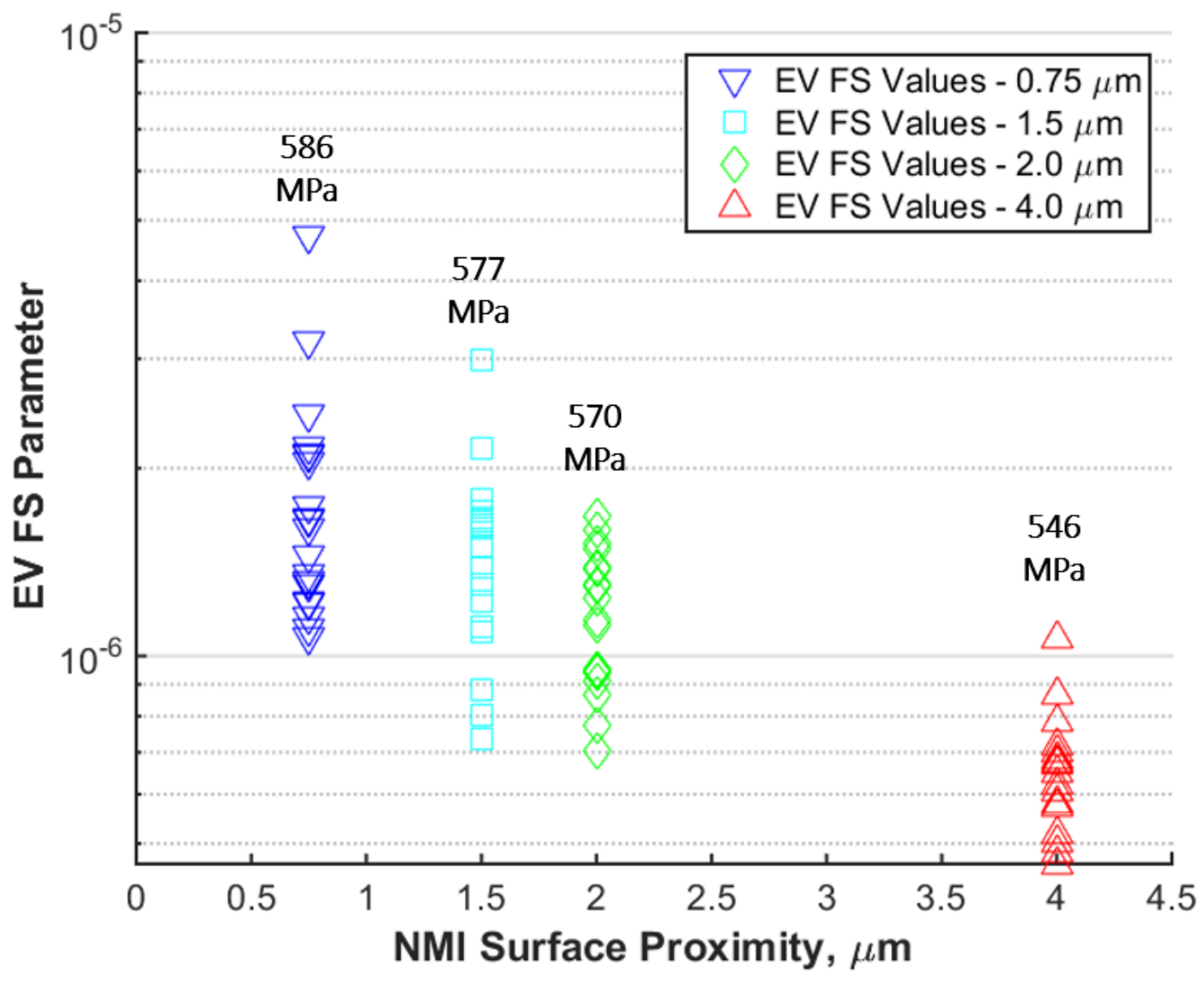

Fig. 4 Extreme-value FS parameter values for four distinct NMI depths and corresponding stress amplitudes reduced proportionally from a nominal $620 \mathrm{MPa}$ stress amplitude at the wire surface. 


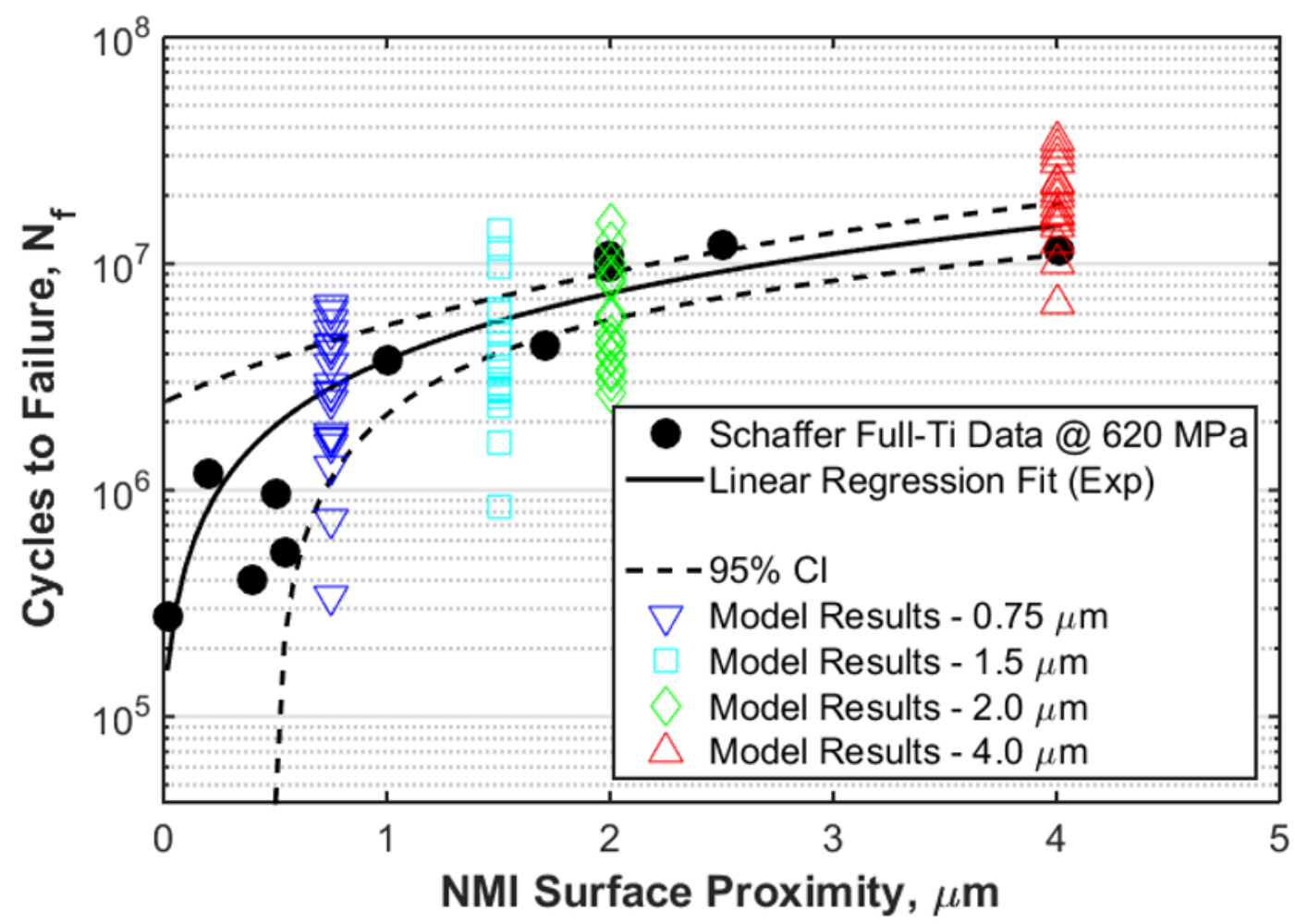

Fig. 5 Life correlations of the model fit to experimental data. The modified T-M fit is performed at the $0.75 \mu \mathrm{m}$ level resulting in a correlation coefficient $\alpha_{g}$ of $1.129 \times 10^{-5} \mu \mathrm{m}$-cycles.

Plotting the CDFs allows for comparison of the probability of failure at a given number of cycles among differing NMI depths from the wire surface. Based on the figure, the probability of failure at $1 \times 10^{7}$ cycles is predicted to be 1.0 for $x_{\text {surf }}=0.75 \mu \mathrm{m}, 0.94$ for $x_{\text {surf }}=$ $1.5 \mu \mathrm{m}, 0.85$ for $x_{\text {surf }}=2.0 \mu \mathrm{m}$ but only 0.07 for $x_{\text {surf }}=4.0 \mu \mathrm{m}$. This result demonstrates that the probability of failure drops off drastically once $x_{\text {surf }}$ reaches or exceeds the diameter of the NMI. This is consistent with past studies [18], which have found that fully embedded NMIs having depth $\left(x_{\text {surf }}\right)$ greater than the diameter of the NMI are associated with substantially lower fatigue potencies than those closer to the surface. The reduction of fatigue potency with increasing depth is enhanced by the stress gradient in RBBF fatigue. These stress gradients are not present in fatigue specimens in tension-compression or tension-tension fatigue. 


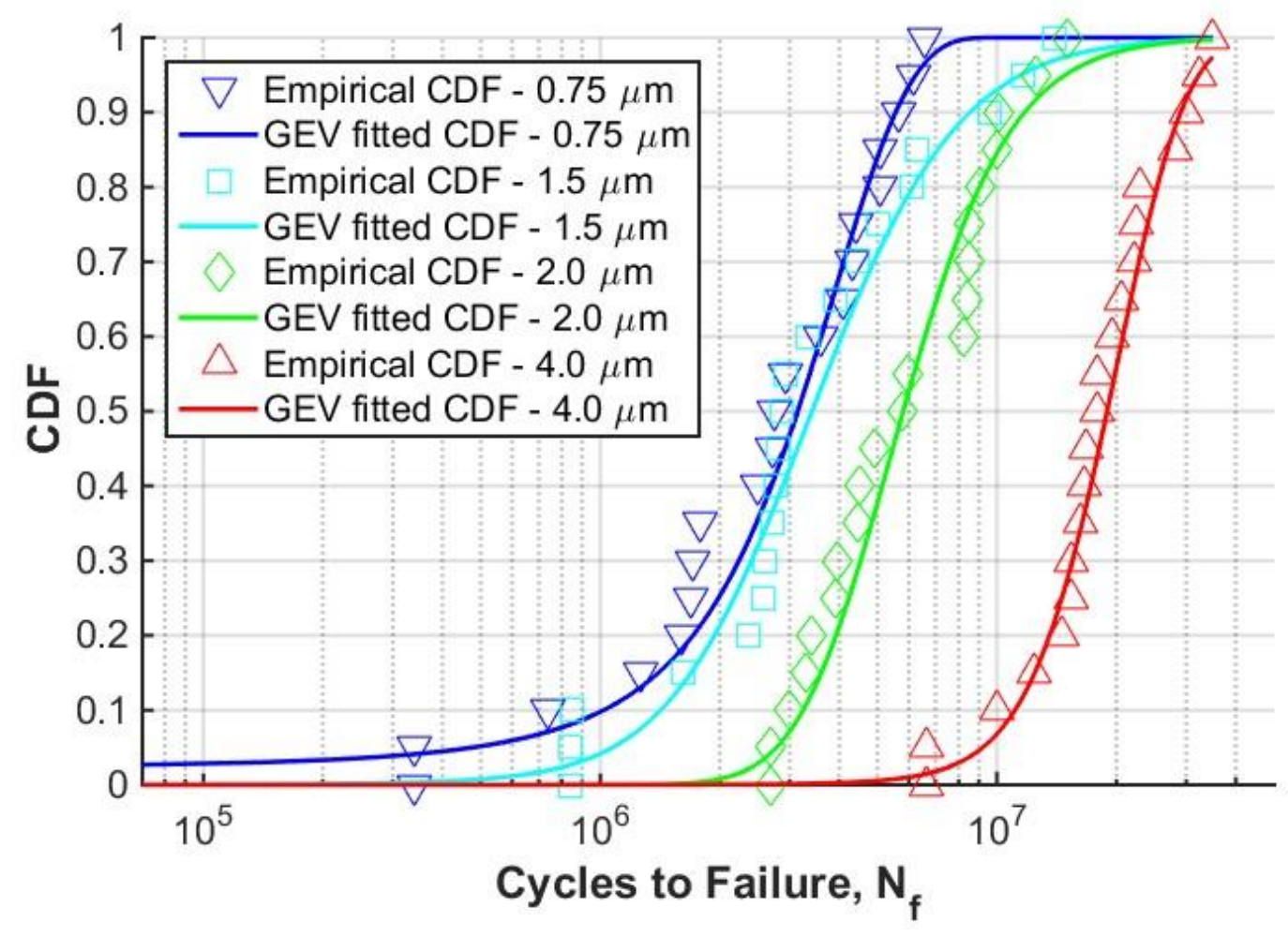

Fig. 6 CDFs of the Fatigue-life correlations with corresponding GEV distributions.

\section{Identifying the Crack Initiation to Microcrack Growth Transition}

A second study was conducted to identify the transition life between fatigue crack initiation and microcrack growth regimes. The transition life value $N_{t}$ can be considered as the value of $N_{f}$ where dominant fatigue mechanism switches from large-scale plasticity and microcrack growth to crack initiation due to highly localized damage accumulation. Because the FS parameter as employed in this model directly equates the localized cyclic shear strain with crack initiation potency most associated with initiation, the transition life $N_{t}$ may be identified as the point of divergence between the experimental mean S-N curve and the model T-M correlation to the initiation regime. At stress amplitudes above this transition, fatigue life is dominated by the cycles to propagate the crack through the wire, with the result that the model 
produces overly conservative life estimates based solely on initiation life.

The experimental fatigue-life data used in this study shown in Fig. 7 was obtained from Prasad et al. [14]. They conducted RBBF on $100 \mu \mathrm{m}$ diameter as-drawn low-Ti MP35N wire at seven stress amplitudes ranging from $1650 \mathrm{MPa}$ (the $0.2 \%$ offset yield strength) down to 550 MPa with $R=-1$. They also performed tension-tension fatigue (TTF) with a stress ratio $R=0.3$ on the same wire. Here, squares represent the RBBF data and circles represent TTF. Filled shapes indicate fractures and open shapes indicate runouts. Note that RBBF tests are displacement controlled, with displacement amplitude being converted to stress amplitude for comparative purposes as described in ASTM E2948-16a [N12]. The displacement controlled nature of RBBF means that the stress intensity factor range, defined as $\Delta K=K_{\max }-K_{\min }$ and the crack growth rate likely decreases as the crack grows due to the increased compliance of the wire with a larger crack size. In contrast, TTF are force-controlled tests, with $\Delta K$ generally increasing with crack growth. Because the increased rate of crack growth as well as the positive mean stress, it is expected that the life to failure in TTF is controlled by crack nucleation at all stress amplitudes and does not exhibit a crack growth dominated regime.

Comparing the RBBF and TTF fatigue data in Fig. 7, it is seen that the TTF life distribution is below that of the RBBF at every comparable stress amplitude. A contributing factor is the difference in the highly-stressed volume due to the test geometry. For equivalent $S_{\max }$, assuming uniform spatial defect distributions, the likelihood of finding a fatigue hotspot with sufficient driving force to nucleate a fatigue crack increases in proportion to the size of the highly-stressed volume. A larger highly-stressed volume samples a much larger subset of the defect population within an individual test specimen and biases the fracture initiation toward higher potency flaws leading to earlier crack nucleation and reduced fatigue life. In addition, the 
likelihood of multiple cracks forming independently and later coalescing into a single large crack increases when the highly stressed volume occupies a significant portion of the overall specimen. In TTF, the entire wire cross section along the entire $255 \mathrm{~mm}$ gage length between the grips is subject to the maximum stress $S_{\max }$. In contrast, only a small portion of the wire near the wire surface and the bend apex approaches $S_{\max }$ in RBBF tests. The highly-stressed volume of the TTF is approximately 133 greater than RBBF, assuming that the highly-stressed volume for $\mathrm{RBBF}$ is an annular cross-section $5 \mu \mathrm{m}$ from the outer wire radius over a length extending $5 \mathrm{~mm}$ on either side of the bend apex. This assumption is based on Schaffer's finding that $90 \%$ of RBBF fractures occur less than $5 \mathrm{~mm}$ from the bend apex and the inclusions that nucleated the fatigue crack are within $5 \mu \mathrm{m}$ of the wire surface [19].

Microstructures for the model correlation were instantiated in the same way as the study described in the last section except that all NMIs were centered in the SVE and fully 3D periodic boundary conditions were prescribed. Ten microstructure instantiations were generated and exposed to five stress amplitudes, 1000, 820, 680, 620 and $550 \mathrm{MPa}$, corresponding to the five lowest stress amplitudes in the Prasad et al. data. No adjustments for the NMI depth from the wire surface were undertaken, instead the far-field stress at the NMI was set equal to the fullyreversed stress amplitude at the wire surface. In other words, each SVE was instantiated with a 4 $\mu \mathrm{m}$ cuboidal inclusion with an applied load commensurate with that at the wire surface, but neglecting the traction-free boundary which was modeled in the prior study. Preliminary modeling showed that influence of changing the alternating stress amplitude on the local FIP response near the inclusion was much larger than the effect of this boundary condition.

The EV FS parameter values are largely unchanged by the choice of $k^{*}$ [N11]. Therefore, the following T-M life correlations use $k^{*}=1$. The T-M correlation of the model EV FS 
response to the Prasad RBBF data was undertaken at the $620 \mathrm{MPa}$ stress amplitude because it was the lowest stress amplitude without runouts found to be dominated by crack nucleation. Using $d_{g}=2 \mu \mathrm{m}$, the correlation coefficient $\alpha_{g}$ was found to be $4.995 \times 10^{-7} \mu \mathrm{m}$-cycles.

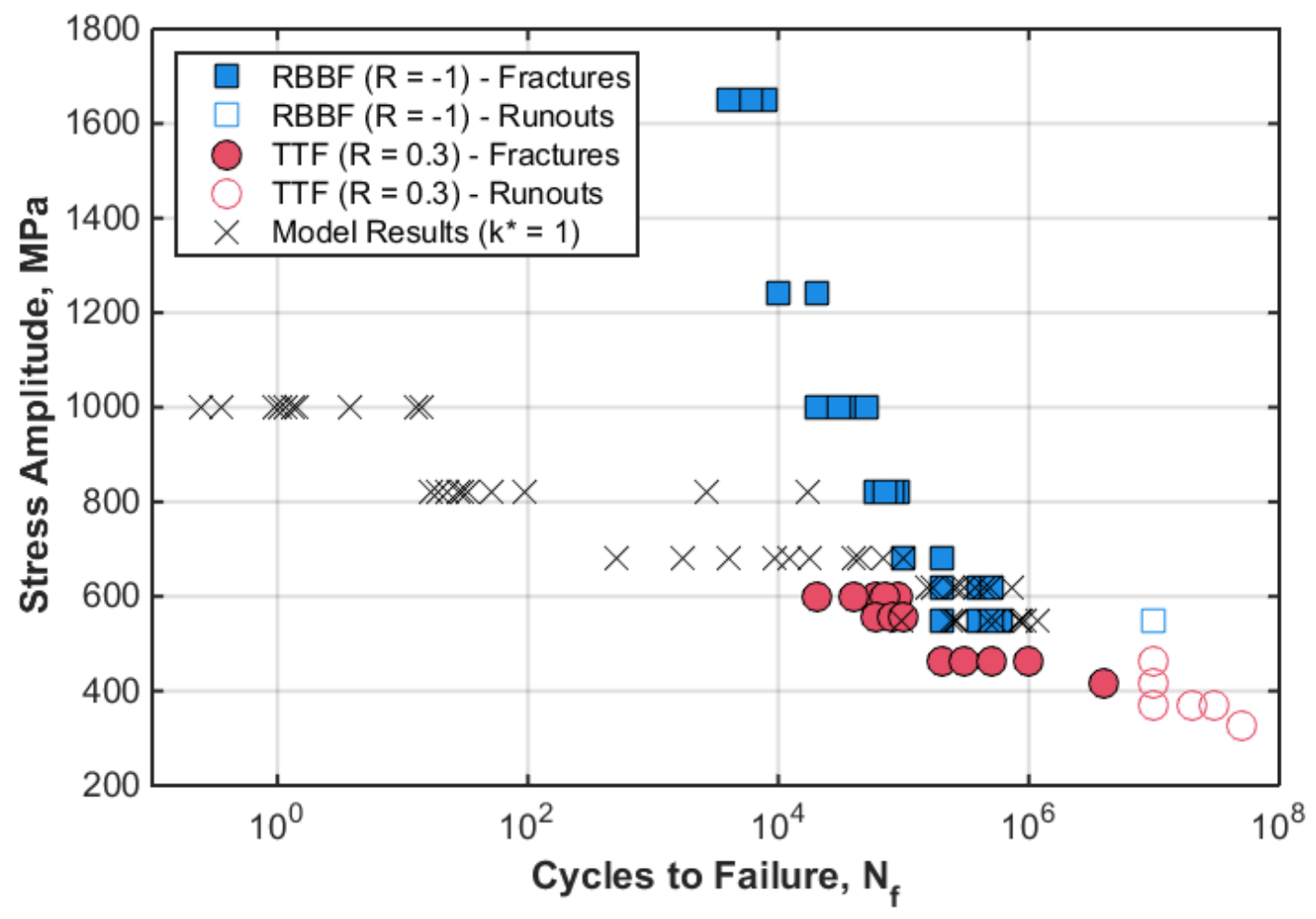

Fig. 7 Life data generated by RBBF and TTF on as-drawn low-Ti MP35N wire [14] and predictions for fatigue crack nucleation correlated to the $620 \mathrm{MPa}$ RBBF data using the T-M model.

Fig. 7 shows the resultant fit with the model results plotted as crosses. The model correlation shows good agreement with RBBF experimental data at $550 \mathrm{MPa}$ but diverges at stress amplitudes higher than $680 \mathrm{MPa}$. Furthermore, the slope from the model is close to that of the TTF experimental data, which further supports the accuracy of our models since we did not use this data to calibrate the model. Above $680 \mathrm{MPa}$, the model predictions are overly conservative compared the $680 \mathrm{MPa} \mathrm{TF}$ experimental data, which suggests that crack propagation indeed actively reduces fatigue life. Thus, we estimate that the transition from crack 
nucleation to propagation for the RBBF data is within the range of life values in the $680 \mathrm{MPa}$ stress amplitude.

The value of $N_{t}$ is very close to $1 \times 10^{5}$ cycles. At life values below $1 \times 10^{5}$ cycles, the T-M model correlation underpredicts the RBBF data suggesting that microcrack growth would dominate the total life to failure. At life values above $1 \times 10^{5}$ cycles, the model correlates well to the RBBF data, indicating that crack nucleation is the main contributor to fatigue life. For RBBF, the stress amplitude of $680 \mathrm{MPa}$ represents the transitional stress where crack nucleation and microcrack growth contribute to the total life in roughly equal measure.

Another indication that the transitional stress amplitude occurs at $680 \mathrm{MPa}$ is the increased scatter of the model predicted lives compared to the other stress levels. This can be seen intuitively by looking at the life correlations in Fig. 7. To quantify the variability at a given stress amplitude, a weighted EV FS variability parameter $\Omega_{F S}$ is defined as

$$
\Omega_{F S}\left(S_{a}, m\right)=\frac{\Delta P_{F S}\left(S_{a}, m\right)}{\tilde{P}_{F S}\left(S_{a}, m\right)}
$$

with $S_{a}$ being the stress amplitude considered and $m$ being the number of instantiations run at that amplitude. The quantity $\Delta P_{F S}$ is the range of EV FS parameter defined as $\Delta P_{F S}=$ $\max \left(P_{F S}\right)-\min \left(P_{F S}\right)$ and $\tilde{P}_{F S}$ is the median of the EV FS values. Weighting the observed scatter by $\tilde{P}_{F S}$ allows for a comparison to be made between FS response parameters spanning several orders of magnitude. Fig. 8 shows a bar graph of $\Omega_{F S}$ for the five stress amplitudes modeled with 10 microstructure instantiations each. The weighted variability at $680 \mathrm{MPa}$ is 5.03 , which is more than twice as large as the next largest value, 1.95, at $1000 \mathrm{MPa}$. Moreover, the value of $\Omega_{F S}$ at $680 \mathrm{MPa}$ is more than three times larger than the values at its neighboring stress amplitudes. The spike in $\Omega_{F S}$ at $680 \mathrm{MPa}$ suggests a heightened sensitivity of the EV FS response to the microstructural features along the NMI debond interface, which can be associated 
with a switch in the dominant fatigue mechanism.

The value of $\Omega_{F S}$ is sensitive to the number of microstructure instantiations run. Since only a small number of microstructure instantiations were run, the values obtained should be treated as a comparative metric only and not representative of the true fatigue variability. Adding additional microstructure instantiations will improve the $\Omega_{F S}$ estimates until the point when EV FS distribution becomes converged.

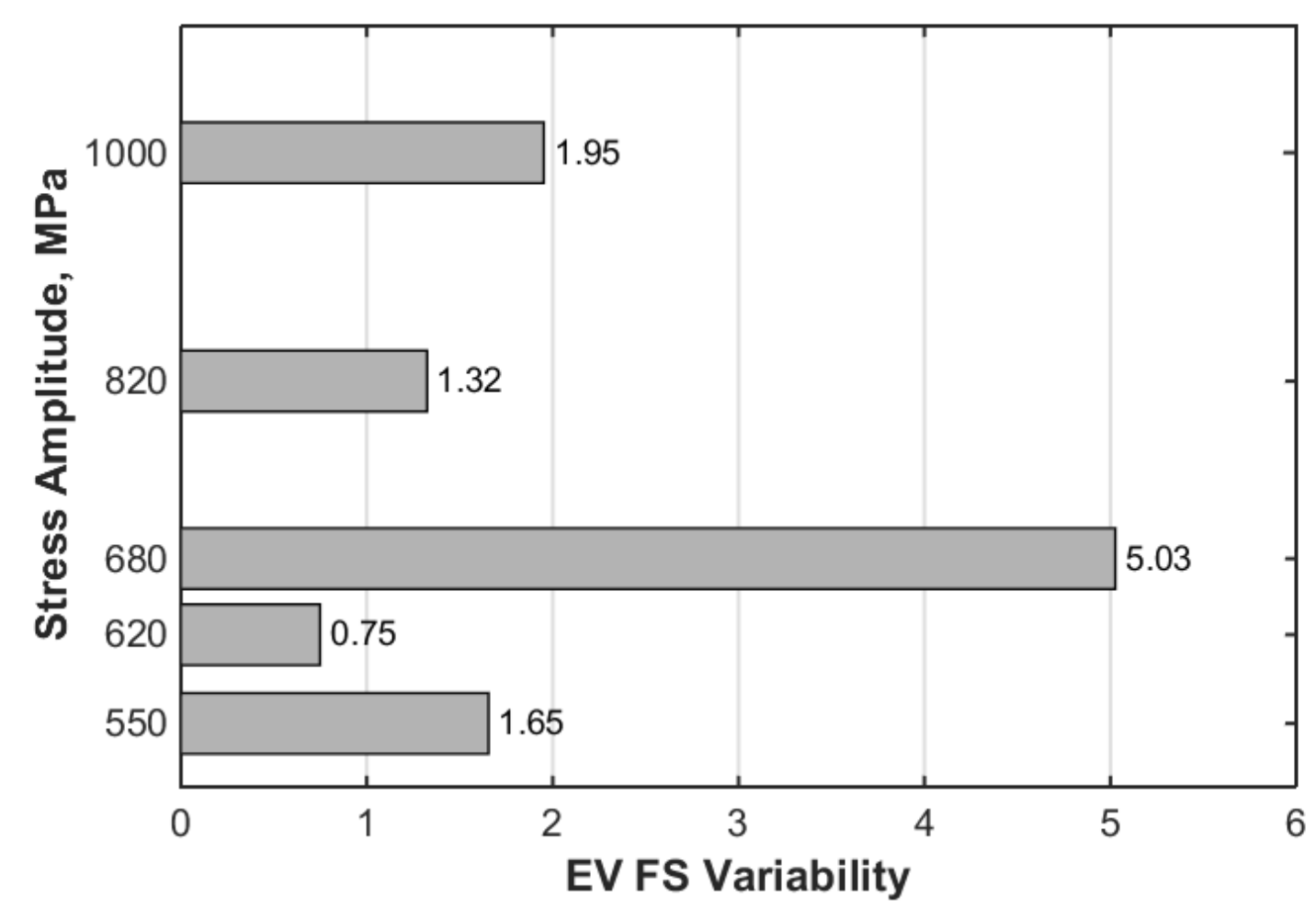

Fig. 8 Weighted variability $\left(\Omega_{F S}\right)$ in EV FS response parameters at the five stress amplitudes modeled with 10 microstructure instantiations each.

\section{SUMMARY AND CONCLUSIONS}

A workflow for establishing the influence of microstructure on the potency for fatigue crack nucleation in fine wire was described and demonstrated. Some key new contributions and observations are summarized. 
A microstructure generation tool was developed to construct statistical volume elements (SVEs) reflecting the fine-grained microstructure and fiber texture characteristic of MP35N fine wire. This included (1) an algorithm for seeding ellipsoidal grains by sampling from a lognormal distribution matched to experimentally characterized grain size distributions, (2) the ability to impose non-random grain texture distributions mimicking the fiber texture, (3) a scheme for placing non-metallic inclusions (NMIs) into SVEs with control over the NMI-matrix interface, and (4) a meshing algorithm to maximize resolution of stress gradients near the NMI while maintaining computational efficiency to run fatigue loading cycles with finite-element solver. By evaluating multiple SVEs, the inherent statistical variability of inclusion-grain and graingrain interactions at the NMI-matrix interface was assessed.

A physically-based, rate-dependent crystal viscoplasticity constitutive model was developed for a single-phase, face-centered cubic MP35N alloy representative of microstructure in the fine wire necessary to model the local cyclic response. Deformation processes at and above the grain scale are modeled, including accumulation of plastic shear strain on preferred slip systems, isotropic and kinematic hardening, and nanoscale twinning through a homogenization approach. The model parameters were fit to a limited number of judiciously chosen mechanical behavior experiments conducted on fine wire, including monotonic strain rate jump and cyclic ratcheting tests.

A modified Tanaka-Mura initiation life relationship was used to correlate the fatigue crack initiation life with the extreme-value Fatemi-Socie parameter volume-averaged over local regions near the NMI-matrix interface. The model correctly predicted the fatigue potency resulting from the variation of NMI proximity to the wire surface conducted in rotating beam bending fatigue (RBBF). A significant reduction in fatigue crack nucleation potency was found 
when NMIs became fully embedded to depth from the surface greater than the diameter of the NMI consistent with fatigue experiments. In another exercise, the transition life between crack initiation and microcrack growth dominated fatigue regimes for fine wire loaded in RBBF was determined. The transition life was estimated to be $1 \times 10^{5}$ cycles based on the point of divergence between the model correlation and the experimental life data which occurred at a stress amplitude of $680 \mathrm{MPa}$, and by the notable increase in the scatter in the predicted lives near this transition. The RBBF data having lives longer than the transition life overlapped the predicted lives, while the experimental RBBF lives less than the transition life were much longer than the predictions, indicating that crack nucleation is no longer the dominant component of the fatigue life, and rather the lives are controlled by microcrack propagation. It is anticipated that the model will effectively correlate the uniaxial tension-tension force-controlled fatigue datasets due to the rapid growth of the microcracks in force-controlled modes of fatigue.

\section{Acknowledgements}

The financial sponsorship of Medtronic, PLC through membership in the NSF (I/UCRC) Center for Computational Materials Design, a joint venture of partner institutions Penn State and Georgia Tech is gratefully acknowledged. The tension-tension cyclic ratchetting response data provided by Jim Hallquist of Medtronic, PLC was critical to the success of the model. The insights from Dr. William Musinski, Dr. Kyle Brindley, and Dr. Ashley Nelson were helpful.

\section{REFERENCES}

[1] Asgari, S., El-Danaf, E., Shaji, E., R., K. S., and Doherty, R. D., "The secondary hardening phenomenon in strain-hardened MP35N alloy," Acta Metallurgica, vol. 46, no. 16, pp. 5795-5806, 1998.

[2] ASTM F562-13, Standard Specification for Wrought 35Cobalt-35Nickel-20Chromium- 
10Molybdenum Alloy for Surgical Implant Applications (UNS R30035), ASTM International, West Conshohocken, PA, 2013, www.astm.org

[3] Castelluccio, G. M. and McDowell, D. L., "Assessment of small fatigue crack growth driving forces in single crystals with and without slip bands," International Journal of Fracture, vol. 176, pp. 49-64, 2012.

[4] Dassault Systemes, “Abaqus v6.14-1,” 2014. Providence, RI, USA.

[5] El-Danaf, E., Kalidindi, S. R., and Doherty, R. D., "Influence of deformation path on the strain hardening behavior and microstructure evolution in low SFE FCC metals," International Journal of Plasticity, vol. 17, pp. 1245-1265, 2001.

[6] Fatemi, A. and Socie, D., "A critical plane approach to multiaxial fatigue damage including out-of-phase loading," Fatigue and Fracture of Engineering Materials and Structures, vol. 11, no. 3, pp. $149-165,1988$.

[7] Li, B. and Steigauf, T., "Crystallography texture and mechanical properties of MP35N wire," in Materials Processes for Medical Devices Conference, ASM, September 2007.

[8] MathWorks, Inc., "Matlab, R2014b,” 2014. Natick, MA, USA.

[9] Musinski, W., Modeling the Effects of Shot-Peened Residual Stresses and Inclusions on Microstructure-sensitive Fatigue of Ni-Base Superalloy Components. $\mathrm{PhD}$ thesis, Georgia Institute of Technology, August 2014.

[10] Musinski, W. D., "Novel methods for microstructure-sensitive probabilistic fatigue notch factor," Master's thesis, Georgia Institute of Technology, August 2010.

[11] Musinski, W. D. and McDowell, D. L., "Microstructure-sensitive proba- bilistic modeling of HCF crack initiation and early crack growth in Ni-base superalloy IN100 notched components," International Journal of Fatigue, vol. 37, pp. 41-53, 2012.

[12] Office of Science and Technology Policy, "Materials genome initiative for global competitiveness," white paper, National Science and Technology Council, June 2011.

[13] Ornberg, A., Pan, J., Herstedt, M., and Leygraf, C., "Corrosion resistance, chemical passivation, and metal release of $35 \mathrm{~N}$ LT and MP35N for biomedical material application," Journal of the Electrochemical Society, vol. 154, no. 9, pp. C546-C551, 2007.

[14] Prasad, M., Reiterer, M., and Kumar, K., "Microstructure and mechanical behavior of an asdrawn MP35N alloy wire," Material Science \& Engineering A, vol. 610, pp. 326-337, 2014.

[15] Przybyla, C. P., Microstructure-sensitive Extreme Value Probabilities of Fatigue in Advanced Engineering Alloys. PhD thesis, Georgia Institute of Technology, 2010.

[16] Przybyla, C. P. and McDowell, D. L., "Simulated microstructure-sensitive extreme value probabilities for high cycle fatigue of duplex Ti-6Al-4V," International Journal of Plasticity, 
2011.

[17] Raghavan, M., Berkowitz, B. J., and Kane, R. D., "A transmission electron microscopic investigation of phase transformations in MP35N," Metallurgical and Materials Transactions A: Physical Metallurgy and Materials Science, vol. 11A, pp. 203-207, 1980.

[18] Salajegheh, N. and McDowell, D. L., "Microstructure-sensitive weighted probability approach for modeling surface to bulk transition of high cycle fatigue failures dominated by primary inclusions," International Journal of Fatigue, vol. 59, pp. 188-199, 2014.

[19] Schaffer, J. E., "A hierarchical initiation mechanism approach to modeling fatigue life variability in $35 \mathrm{Co}-35 \mathrm{Ni}-20 \mathrm{Cr}-10 \mathrm{Mo}$ alloy medical grade fine wire," Master's thesis, Purdue University, 2007.

[20] Schaffer, J. E., "An examination of fatigue initiation mechanism in thin $35 \mathrm{Co}-35 \mathrm{Ni}-20 \mathrm{Cr}-$ 10Mo medical grade wires," Journal of ASTM International, vol. 5, pp. 1-10, July 2008.

[21] Shenoy, M., Tjiptowidjojo, Y., and McDowell, D. M., "Microstructure-sensitive modeling of polycrystalline IN 100," International Journal of Plasticity, vol. 24, pp. 1694-1730, 2008.

[22] Shenoy, M., Zhang, J., and McDowell, D. L., "Estimating fatigue sensitivity to polycrystalline Ni-base superalloy microstructures using a computational approach," Fatigue and Fracture of Engineering Materials and Structures, vol. 30, pp. 889-904, 2007.

[23] Sorensen, D., Li, B., Gerberich, W., and Mkyoyan, K., "Investigation of secondary hardening in Co-35Ni-20Cr-10Mo alloy using analytical scanning transmission microscopy," Acta Materialia, 2013.

[24] Suresh, S., Fatigue of Materials. Cambridge University Press, $2^{\text {nd }}$ Ed., 1998.

[25] Tanaka, K. and Mura, T., "A dislocation model for fatigue crack initiation," Journal of Applied Mechanics, vol. 48, pp. 97-103, 1981.

[26] Wang, Y., Wang, J. J., Zhang, H., Manga, V. R., Shang, S. L., Chen, L.-Q., and Liu, Z.-K., "A first-principles approach to finite temperature elastic constants," Journal of Physics: Condensed Matter, vol. 22, pp. 225404-225412, 2010.

[N1] McDowell, D. L. (2007). Simulation-based strategies for microstructure-sensitive fatigue modeling. Materials Science and Engineering A, 468-470, 4-14.

[N2] McDowell, D. L., \& Dunne, F. P. E. (2010). Microstructure-sensitive computational modeling of fatigue crack formation. International Journal of Fatigue, 32, 1521-1542.

[N3] Przybyla, C., Prasannavenkatesan, R., Salajegheh, N., \& McDowell, D. L. (2010). Microstructure-sensitive modeling of high cycle fatigue. International Journal of Fatigue, 32(3), $512-525$.

[N4] Przybyla, C. P., Musinski, W. D., Castelluccio, G. M., \& McDowell, D. L. (2013). 
Microstructure-sensitive HCF and VHCF simulations. International Journal of Fatigue, 57(0), 927. doi:http://dx.doi.org/10.1016/j.ijfatigue.2012.09.014

[N5] Przybyla, C. P., \& McDowell, D. L. (2010). Microstructure-sensitive extreme value probabilities for high cycle fatigue of Ni-base superalloy IN100. International Journal of Plasticity, 26, 372-394. doi:10.1016/j.ijplas.2009.08.001

[N6] Prasannavenkatesan, R., Przybyla, C. P., Salajegheh, N., \& McDowell, D. L. (2011). Simulated extreme value fatigue sensitivity to inclusions and pores in martensitic gear steels. Engineering Fracture $\quad$ Mechanics, $\quad 78(6), \quad 1140-1155$. doi:https://doi.org/10.1016/j.engfracmech.2011.01.027

[N7] Przybyla, C. P., \& McDowell, D. L. (2012). Microstructure-sensitive extreme-value probabilities of high-cycle fatigue for surface vs. subsurface crack formation in duplex Ti-6Al4V. Acta Materialia, 60(1), 293-305. doi:10.1016/j.actamat.2011.09.031

[N8] Musinski, W. D., \& McDowell, D. L. (2016). Simulating the effect of grain boundaries on microstructurally small fatigue crack growth from a focused ion beam notch through a threedimensional array of grains. Acta Materialia, 112, 20-39. doi:https://doi.org/10.1016/j.actamat.2016.04.006

[N9] Swaminathan S, Ghosh S, Pagano NJ. Statistically equivalent representative volume elements for unidirectional composite microstructures: part I - Without damage. J Compos Mater 2006;40:583-604.

[N10] Swaminathan S, Ghosh S. Statistically equivalent representative volume elements for unidirectional composite microstructures: part II - With interfacial debonding. J Compos Mater 2006;40:605-21.

[N11] Clark, B.C., 2016, Microstructure-sensitive fatigue modeling of medical-grade fine wire, M.S. Thesis, Woodruff School of Mechanical Engineering, Georgia Institute of Technology, Atlanta, GA.

[N12] ASTM E2948-16a, Standard Test Method for Conducting Rotating Bending Fatigue Tests of Solid Round Fine Wire, ASTM International, West Conshohocken, PA, 2016, www.astm.org

[GMC1] Castelluccio, Gustavo M., and David L. McDowell. "Microstructure-Sensitive Small Fatigue Crack Growth Assessment: Effect of Strain Ratio, Multiaxial Strain State, and Geometric Discontinuities." International Journal of Fatigue 82, Part 3 (January 2016): 521-29. doi:10.1016/j.ijfatigue.2015.09.007.

[GMC2] Castelluccio, Gustavo M., and David L. McDowell. "Microstructure and Mesh Sensitivities of Mesoscale Surrogate Driving Force Measures for Transgranular Fatigue Cracks in Polycrystals." Materials Science and Engineering: A 639 (July 15, 2015): 626-39. doi:10.1016/j.msea.2015.05.048. 
[GMC3] Gustavo M. Castelluccio, David L. McDowell, Mesoscale cyclic crystal plasticity with dislocation substructures, International Journal of Plasticity, In press June 2017, ISSN 07496419, https://doi.org/10.1016/j.ijplas.2017.06.002

[GMC4] Castelluccio, Gustavo M., and David McDowell. "Assessment of Small Fatigue Crack Growth Driving Forces in Single Crystals with and without Slip Bands." International Journal of Fracture 176, no. 1 (July 1, 2012): 49-64. doi:10.1007/s10704-012-9726-y.

[GMC5] Castelluccio, Gustavo M., and David L. McDowell. "A Mesoscale Approach for Growth of 3D Microstructurally Small Fatigue Cracks in Polycrystals." International Journal of Damage Mechanics 23, no. 6 (2014): 1056789513513916. doi:10.1177/1056789513513916. 
2018-06-27

\section{Microstructure-sensitive fatigue modelling of medical-grade fine wire}

Clark, B. C.

Wiley

Clark BC, Catelluccio GM, Reiterer M, McDowell DL, Neu RW, Microstructure-sensitive fatigue modeling of medical-grade fine wire, Fatigue \& Fracture of Engineering Materials \& Structures, Volume 42, Issue 1, January 2019, pp. 152-165

http://dx.doi.org/10.1111/ffe.12879

Downloaded from Cranfield Library Services E-Repository 